\title{
2010 International Forum on Stem Cells (November 11-13; Tianjin, China)
}

\author{
Douglas Sipp • Zack Wang • Weiping Yuan
}

Published online: 2 March 2011

(C) Springer Science+Business Media, LLC 2011

More than 500 scientists and students gathered in Tianjin, China for a two-day meeting on the latest advances in stem cell research and applications, ranging from fundamental studies of pluripotency to lineage commitment, to the role of stem cell in oncological diseases. Sponsored by the Chinese Academy of Medical Sciences (CAMS) and the National Natural Science Foundation of China (NNSF), and co-chaired by Tao Cheng of the Center for Stem Cell Medicine and Institute of Hematology at CAMS (Tianjin, China), Toshio Suda of Keio University (Tokyo, Japan) and Armand Keating of University of Toronto (Toronto, Canada), the program of the $\mathbf{2 0 1 0}$ International Forum on Stem Cells (IFSC), which included multiple plenary and concurrent sessions, poster sessions, and a round table discussion, brought together some of the world's leading authorities in stem cell science. Stem Cell Reviews and Reports supported the meeting and participated actively as well, as part of its ongoing commitment to fostering excellence in the field in China and throughout the Asia region. With more than 50 speakers, it would be impossible to do justice to all in the short format of this report, but we

\footnotetext{
D. Sipp $(\bowtie)$

RIKEN Center for Developmental Biology,

2-2-3 Minatojima Minamimachi,

Kobe, Japan 650-0047

e-mail: sipp@cdb.riken.jp

Z. Wang

Center for Molecular Medicine, Maine

Medical Center Research Institute,

81 Research Drive,

Scarborough, ME 04074, USA

W. Yuan

State Key Laboratory of Experimental Hematology,

288 Nanjing Road,

Tianjin, People's Republic of China
}

hope to provide a glimpse of the spectrum of topics covered in the Forum's program.

Irv Weissman of Stanford University opened the forum with a plenary talk reviewing one thread of work by his work during his distinguished career in stem cell research, describing the approaches to hematopoietic stem cell-based applications for the treatment of cancer. After introducing a number of highlights from the history of hematopoietic stem cell (HSC) research, Weissman spoke on how the use of monoclonal antibody against c-Kit can condition hosts for congenic engraftment, and prevent graft versus host responses by blocking c-Kit/SCF signaling.

He next explained a technique for rendering leukemia stem cells visible to the immune system through the inhibition of CD47, which he described as an "invisibility cloak for cancers," which allows them to evade phagocytosis. Work on the combinatorial use of rituximab and an anti-CD47 antibody showed very high survival of mice with human tumors. CD47 is expressed in a wide range of cancer cells and the Weissman group is now seeking to develop an antibody for clinical use. His work highlights the importance of reliable methods for identifying and screening individual cell types when developing cellular therapeutics.

Edward Srour of Indiana University spoke on the cellular components of the HSC niche provides the diverse aggregation of cells in the bone marrow. His team has developed 2- and 3D culture systems for studying integrated bone marrow environments and showed that co-culture of stromal cells with osteoblasts at high densities promotes the formation of colony forming units (CFUs), reconfirming the importance of these cells to the HSC niche. He next reviewed his work on stages of osteoblast maturation, which showed that less mature cells, as characterized by higher expression levels of Runx-2, were better able to support stem cell self-renewal. 
In his talk, Gerald de Haan of the University of Groningen asked whether the HSC aging is a programmed process, or simply the result of genetic noise. Studying chimeric mice generating by crossing $\mathrm{C} 5 \mathrm{BL} / 6$ (which live long, and have slowly cycling stem cells) and DBA/2 (which show the opposite phenotype) mice, his group found that DBA/2 cells were lost over time. Checking for differences in gene expression and epigenetics, they identified a number of genes with twofold or greater transcriptional changes, as well as altered chromatin states. Older HSCs showed greater variability in their transcriptomes, with a pair of $\mathrm{Cbx}$ genes, $\mathrm{Cbx} 7$ and 8 , being of special interest. Overexpression of these genes revealed opposing effects, with $C b x 7$ triggering leukemogenesis, while $\mathrm{Cbx} 8$ led to competitive disadvantage for cells in vivo. De Haan presented a model in which the HSC sits atop a steeply sloping hill of homeostasis, with genes associated with marrow failure to one side, and those promoting leukemia on the other.

Speaking on the issues confronting the clinical translation of stem cell research, Armand Keating outlined many of the challenges investigators will face to bring their work from the lab to therapeutic applications. He stressed the need for good animal models in preclinical testing and standardization of products used in cell formulations. He further emphasized the need for proper study design, and core operational funding to sustain costly translational research through an ever-widening "valley of death."

Sheng Ding of Scripps Research Institute described a chemical approach to the controlling cellular fates involving lead optimization and screening of small molecules capable of maintaining stem cells or inducing their differentiation. His synthetic approach has been famously successful in identifying small molecule replacements for transcription factors used in reprogramming events such as nuclear transfer and the generation of induced pluripotent stem cells (iPSCs). His team introduced a new molecule that enables iPSC generation using only the single transcription factor Oct4, as well as two new synthetic molecules that promote the survival of human embryonic stem cells (hESCs) through inhibition of ROCK, thereby stabilizing hESC cell-cell junctions.

A further look at the HSC niche was provided by Toshio Suda, who described telltale differences in gene expression between mature osteoblasts and multipotent progenitor cells (MPCs), showing that while cell adhesion genes are upregulated in osteoblasts, MPCs tend to express cytokine-related genes. Osteoblasts have been shown to reside in a vascular/hypoxic niche and express HIF-1 $\alpha$; a conditional HIF-1 $\alpha$ knockout resulted in loss of quiescent HSCs, accelerated cell cycle and increased expression of cycle-related genes, resulting in poor engraftment and repopulation on transplantation. A second line of work focused on F-box WD-40 protein 7 (FBxw7), a ubiquitin ligase that maintains HSCs by mediating degradation of cell cycle activators. FBxw7 loss of function can lead to leukemogenesis, presumably through accumulation of oncogenes such as c-Myc. Overexpression of Fbxw7 suppresses Notch signaling, and its upregulation is triggered by hypoxia. Given these findings, Suda suggests that radical oxygen species may be the stem cells' Achilles heel, causing them to differentiate more rapidly, leading to stem cell exhaustion and ultimately bone marrow failure.

Linheng $\mathbf{L i}$ of the Stowers Institute examined how active and quiescent cells can coexist in a single system, using the intestinal crypt as a model. The zonal structure of the crypt is well-defined, with an active crypt-based columnar cell (CBC) expressing Lgr5 at the bottom-most position, and quiescent label-retaining cells (LRCs) located at the +4 . $\mathrm{Li}$ explained how when the $\mathrm{Lgr}^{+}$population is killed by a lethal dose of irradiation, the same gene is upregulated in other radiation-resistant non-cycling cells, which then initiate regeneration. The determination of activity or quiescence seems to be under the control of the Wnt and BMP pathways, with Wnt off and BMP on in the inhibitory zone, and the opposite relationship in the stimulatory zone in the gut.

The role of stem cells in cancer, and their viability as a target for novel anti-cancer treatments is one of the hottest questions in the field, and Shaoguang Li of the University of Massachusetts reviewed his work in identifying the critical pathways operating in cancer stem cells. He conducted an optimized DNA microarray analysis on normal HSCs and leukemic stem cells (LSCs) and identified around 20 genes showing marked differences in expression level. Among these, one gene of particular interest was Alox5, a component of a common lipid metabolism pathway. Mice engineered to lack this gene showed resistance to chronic myeloid leukemia induced by BCR-ABL, due to impaired differentiation, cell cycle and survival of LSCs. Li further described a tumor-suppressing role for stearoyl CoA desaturase (Scd1); induction of its encoding gene by rosiglitazone inhibited leukemic stem cell function. Similarly, a third factor, Msr1 (Macrophage scavenger receptor 1), which is upregulated in CML in the absence of Alox5, can also function as a tumor suppressor through the regulation of apoptosis.

Ting Xie of the Stowers Institute started his talk with a comprehensive overview of his and others' work in the characterization of the germline stem cell niche in the Drosophila ovary, noting the niche-maintaining role of Notch signaling in this context. He then switched focus from basic science to application, giving a look at the potential for retinal stem cells in the treatment of degenerative diseases of the eye. His team was able to culture single cells from adult mouse retina and character- 
ized them as positive for Nestin, Pax6, and Sox2. These cells can give rise to all types of cell in the retina, and can be induced at efficiencies of $30-40 \%$ to differentiate into retinal ganglion cells, which will integrate into the appropriate layer on injection into a glaucomic mouse eye. Photoreceptors differentiated from these retinal stem cells also integrate and form synapses on transplantation, highlighting the potential of this technology for regenerative medicine.

Diabetes is reaching epidemic levels worldwide, and now affects as many as $10 \%$ of the Chinese population, with another 15\% considered pre-diabetic. Hongkui Deng of Peking University described his work toward generating functional pancreatic beta cells for possible therapeutic use. His team has previously generated insulin producing cells from mouse and human ESCs, as well as human iPSCs, using a stepwise model moving from stem cell to definitive endoderm, to pancreatic progenitor, to beta cell at efficiencies well over $80 \%$. He is also interested in alternative approaches such as the replication of pre-existing beta cells, and direct reprogramming. After finding that beta cell replication can be inhibited by the overexpression of $\mathrm{p} 21$, he used this to develop a model for testing whether nonbeta cells can differentiate into insulin-producing cells after the majority of beta cells are lost. His group discovered that when mice overexpressing p21 are administered streptozotocin to induce diabetes, they showed better recovery than control and developed large proliferative populations of $\mathrm{PDX}^{+}$cells, suggesting that the non-beta islet population gives rise to pancreatic progenitors on $\mathrm{p} 21$ depletion.

Linzhao Cheng of Johns Hopkins University described his recent work generating human iPSCs from blood, which provides an accessible source of cells and extensive banked resources, as well as around a dozen acquired blood-only disorders that might benefit from stem cell resources. His team succeeded in generating iPSCs from CD34+ cells using the four Yamanaka factors, Oct4, Sox2, Klf4 and cMyc, delivered by retroviral vector. Not satisfied with the conventional method, they have tested a number of alternative delivery systems (episomal vectors) and source cells (cord blood), which yielded genetically and karyotypically stable iPSCs at (in the case of cord blood) efficiencies as high as $0.1 \%$. The group now holds seven characterized iPS cell lines.
The Forum closed with a talk by Douglas Sipp of the RIKEN Center for Developmental Biology on global issues surrounding the commercialization of unproven stem cell treatments. After giving an overview of the growth of this industry in various countries, he illustrated how companies marketing untested medical uses of stem cells are increasingly advertising such treatments using practices common to alternative or holistic medicine.

In addition to the numerous plenary talks described above, the meeting featured multiple concurrent sessions on such themes as pluripotency and development, therapeutics, hemogenesis, tissue engineering, cardiovascular differentiation and immune regulation, HSCs, neural and skin differentiation, and stem cells in disease. These sessions provided opportunities for 40 scientists from China and abroad to introduce their work in short-format talks, followed by intensive discussion.

The 2010 IFSC was a success by any measure, allowing colleagues from around the world to discuss their own work and gain insights into advances from other areas of the rapidly diversifying field of stem cell research. The high quality research presented at the meeting also provided young inspiring scientists and graduate students in China a taste of the frontiers of stem cell research, and one which may have a real impact on their future career pathways. Prior to the forum, a training course on stem cell technologies, primarily taught by the Chinese faculty speakers from the States, was also held, giving young participants an opportunity to learn a number of cuttingedge methods.

Moreover, the Forum served as a wonderful introduction to stem cell research activities in the city of Tianjin, whose Center for Stem Cell Medicine and Institute of Hematology at the CAMS and the State Key Laboratory of Experimental Hematology played central roles in organizing the program and hosting the event. A number of speakers also toured the nearby institutes, including Union Stem Cell and Gene Co., the world's largest cord blood bank, and Nankai University. The excellent fundamental science being done by labs from around China was very much in evidence in both the oral and poster sessions, and stands as a testament to the Chinese governments' increasing investments into basic and applied aspects of stem cell research, and to the talent and dedication of the scientists who work there. 